\title{
STUDY OF SERUM LACTATE LEVELS IN PATIENTS WITH SEPSIS ON THE BASIS OF SURVIVAL AND NON-SURVIVAL
}

KEY WORDS: Lactate, Sepsis, Mortality, Septic-shock, Severe sepsis

\section{Anisha Tanwar}

\section{Bushra Fiza}

\section{Bhupesh} Medatwal

\section{Rubal Singh*}

\section{Maheep Sinha}

Department Of Biochemistry, Mahatma Gandhi Medical College And Hospital (M.S.C. (Medical) scholar)

Department Of Biochemistry, Mahatma Gandhi Medical College And Hospital (Professor)

\section{Department Of Biochemistry, Mahatma Gandhi Medical College And Hospital} (Demonstrator)

Department Of Biochemistry, Mahatma Gandhi Medical College And Hospital (Ph.D. Scholar). *Corresponding Author

Department Of Biochemistry, Mahatma Gandhi Medical College And Hospital (Professor \& Head)

\footnotetext{
INTRODUCTION:- Sepsis is a leading cause of morbidity and mortality internationally. Early spotting and interventions are essential to ensure better patient's outcome. Lactate concentration in septic patients is of particular prognostic value in predicting septic shock and mortality.

AIM:- The present study was intended to evaluate the serum Lactate levels on the basis of severity rate in patients

suffering of sepsis, severe sepsis and septic shock.

MATERIAL \& METHOD:- 100 patients diagnosed for sepsis were enrolled for the study and they were grouped as sepsis $(n=30)$, severe sepsis $(n=37)$ and septic shock $(n=33)$. On the basis of survival rate serum Lactate levels in patients with sepsis were estimated in all the 3 categories of subjects according to the inclusion criteria. A p-Value of $\leq 0.05$ were considered as statistically significant.

RESULT:- In the present study when the lactate levels were evaluated among all the three categories, it was observed that the Lactate levels were found to be statistically significant $(p$-value $<0.0001)$. Lactic acid evaluated on the basis of survival rate was also found to be statistically significant with the $p$-Value 0.015 .

CONCLUSION:- Lactic acid could be utilized as a reliable marker to assess prognosis at the initial phase of presentation because its initial evaluation shows good predictability in mortality prediction in sepsis patients.
}

\section{INTRODUCTION}

Sepsis is a systemic inflammatory response syndrome that affects all organ systems. It is a potentially fatal disease resulting from an uncontrolled host response to infection and is a major cause of morbidity and mortality worldwide. Sepsis covers a wide range of illnesses, from mild signs and symptoms to organ dysfunction (severe sepsis) and shock. (1) Accurately identifying sepsis is a challenge in emergency care. Despite advances in antibiotic therapy and modern life support, mortality in patients with sepsis remains high worldwide (by $>30 \%$ ). $(2,3$ ) Sepsis occurs approximately $2 \%$ of all inpatients in developed countries and $6 \%$ to $30 \%$ of all patients in the intensive care unit (ICU), with large variations due to heterogeneity between ICUs. (4) Early identification of patients at high risk of death from sepsis can help initiate timely and appropriate therapeutic intervention. (5)

Biomarkers can help in early diagnosis, evaluation of severity, prognosis, guiding antibiotic therapy. They can also help in evaluating the response to therapy and recovery from sepsis, differentiating infectious and non-infectious causes of systematic inflammatory response syndrome (SIRS). Likely it's having a role in predicting sepsis complications and the development of organ down regulation. (6) Lactic acid being known as a product of glycolysis during hypoxia. (7) Lactic acidosis (accumulation of lactic acid and protons in the body fluid) is often related with poor clinical outcomes. (8)

In normal physiologic conditions, blood lactate levels are typically between $0.5-1 \mathrm{mmol} / \mathrm{L}$. However, in critically ill patients, persistent levels $>2 \mathrm{mmol} / \mathrm{L}$. This level is indicator of poor tissue perfusion and worse patient outcome. (9) Increased level of lactic acid (Hyperlactatemia) in sepsis and septic shock occurs as a result of tissue hypoxia. (10)
Some causes of Lactic acidosis are seizures, exercise, shivering but the body clears Lactate quickly, thus significant serum levels does not occur. (11) So, the lactate level is usually elevated in severe sepsis and septic shock due to organ dysfunction. (12) It is recommended by the 'Surviving sepsis' campaign that the lactate levels estimation at the time of admission plays very important role in deciding the management of sepsis patients. (13)

Lactic acid is widely used as a marker of tissue perfusion in critically ill patients. Under normal physiological conditions, blood lactate levels are generally within $0.5 \mathrm{mmol} / \mathrm{L}$ produced by anaerobic metabolism in various organs and tissues. (9) However, in critically ill patients, sustained lactate levels above $2 \mathrm{mmol} / \mathrm{L}$ indicate inadequate tissue perfusion and poor patient outcomes. Patients with lactate levels above $4 \mathrm{mmol} / \mathrm{L}$ are at particular risk of death regardless of shock. (14) Previous studies have indicated that lactate levels have particular prognostic value in predicting septic shock and mortality in septic patients. Based on this work, clinicians have implemented lactate standardized goals for the management of sepsis patients through a sepsis care package, consistent with the Sepsis Surviving guidelines. (13)

Therefore, this study is designed to assess the role of blood lactate in patients with sepsis.

\section{MATERIALS AND METHODS}

The study was conducted in Department of Biochemistry in association with Department of General Medicine of Mahatma Gandhi Medical College \& Hospital, Jaipur, Rajasthan.

A study protocol was approved by the Institutional Ethics Committee (IEC) and informed consent was taken before 
enrolling the patients for the study. Study population consists of total 100 Patients diagnosed for SEPSIS, visiting the Inpatient Department (IPD) of General Medicine fulfilling the inclusion criteria were enrolled for the study. Patients were grouped on the basis of $\operatorname{sepsis}(n=30)$, severe $\operatorname{sepsis}(n=37)$ and septic shock $(\mathrm{n}=33)$ categories.

Inclusion Criteria for the study: Age between 15- 65 years, either gender, patients diagnosed with sepsis, patients who willingly participated and signed consent document.

Exclusion Criteria for the study: Patients with co-morbidities of severe liver dysfunction, cardio and cerebrovascular disease, blood system disease, renal transplant therapy, malignant tumor, diabetes, pregnant and lactating women.

Blood samples for all subjects were collected using standard aseptic technique and analyzed for serum lactate level (By Lactate oxidase method- colorimetry on VITROS 5600 autoanalyzer). The results obtained during the study were presented as mean $\pm \mathrm{SD}$. The result of lactate in above subgroups were assessed by applying one way anova. pvalue $\leq 0.05$ was considered statistically significant.

\section{RESULT}

In the present study 100 patients were enrolled fulfilling inclusion criteria among which sepsis patients were 30 , severe sepsis were 37 and septic shock were 33 . When cases were distributed on the basis of age, majority of cases (47\%) were above the age of 45 years and $35 \%$ of cases were in the age group of $25-45$ years and $31 \%$ cases were above the age of 55 years. The male: female ratio was 3.1:1, showed that the percentage of males affected with sepsis was higher as compared to females. Overall mortality was $20 \%$ among nonsurvival group, in which $10 \%$ cases were in sepsis group, $16.22 \%$ and $33.33 \%$ were in severe sepsis and septic shock group respectively ( $\mathrm{p}$-value-0.006).

The mean age of total population was $42.57 \pm 14.77$. The mean age of survivors was $41.96 \pm 15.49$ and non-survivors was $45.33 \pm 10.88$ and it was non-significant. Normal biological reference range of blood lactate is between $0.5-1.0 \mathrm{mmol} / \mathrm{L}$.

Distribution of cases on the basis of blood lactate showed Mean \pm SD for sepsis group, severe sepsis and septic shock group were $1.00 \pm 0.60,3.29 \pm 0.51,10.79 \pm 2.82$ respectively (pvalue $<0.0001$ ) as shown in table no.l, and is graphically presented as figure no.l.

Table 1: Distribution Of Cases On The Basis Of Blood Lactate Level.

\begin{tabular}{|c|c|c|c|c|c|}
\hline Parameter & Sepsis & $\begin{array}{c}\text { Severe } \\
\text { sepsis }\end{array}$ & $\begin{array}{c}\text { Septic } \\
\text { shock }\end{array}$ & $\begin{array}{c}\text { F- } \\
\text { value }\end{array}$ & $\begin{array}{c}\text { p- } \\
\text { value }\end{array}$ \\
\hline Blood lactate & $1.00 \pm$ & $3.29 \pm$ & $10.79 \pm$ & 299. & $<0.000$ \\
level (mmol/L) & 0.60 & 0.51 & 2.82 & 52 & 1 \\
\hline
\end{tabular}

$P$-value as obtained on applying One way ANOVA

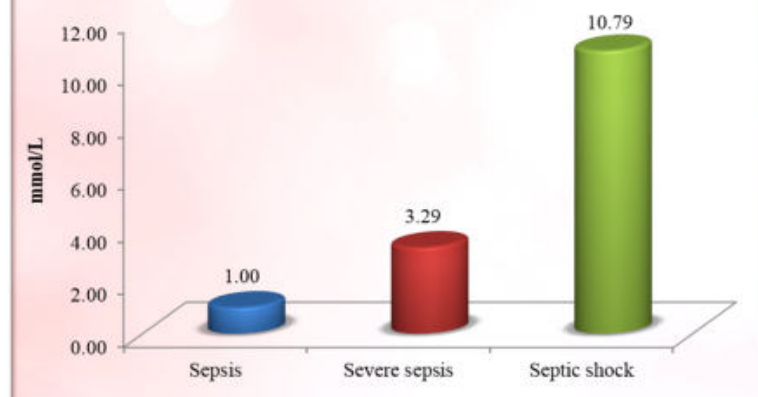

Figure 1: Distribution of cases on the basis of Blood Lactate Level.

www.worldwidejournals.com
Mean value of Lactate when distributed on the basis of survival, in survivors it was $3.10 \pm 1.59$ and in non-survivors $4.20 \pm 4.56$ respectively. It was statistically significant (pValue- 0.015 ) as shown in table 2 and graphically presented as figure 2 .

Table 2: Distribution on the basis of Survival and NonSurvival Lactate Mean Value

\begin{tabular}{|c|c|c|c|c|}
\hline Parameters & Survival & Non-Survival & t-value P-value \\
\hline $\begin{array}{c}\text { LACTATE } \\
\text { (mmol/L) }\end{array}$ & $3.10 \pm 1.59$ & $4.20 \pm 4.56$ & -2.472 & 0.015 \\
\hline
\end{tabular}

P-value as obtained on applying student's t-test

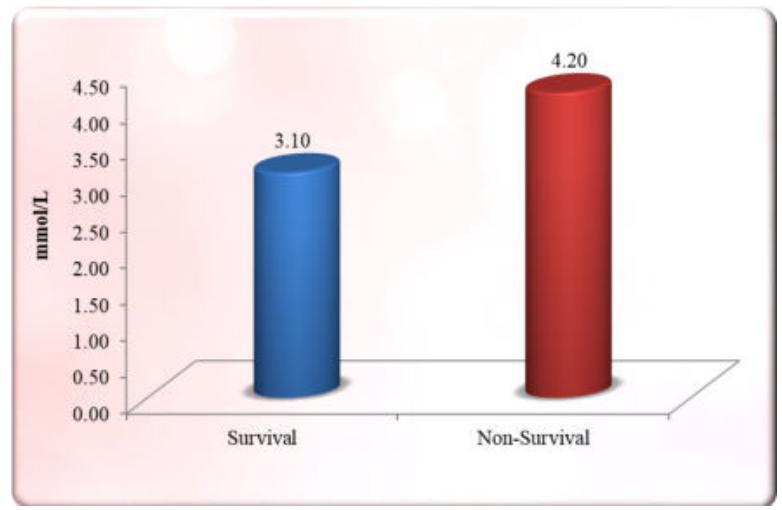

Figure 2: Distribution on the basis of Survival and Non survival Lactate IMean Value

\section{DISCUSSION}

The present study was conducted to explore the importance of Blood Lactate levels in patients of sepsis and to evaluate its importance in survival and non-survival of sepsis patients. When cases were distributed on the basis of age, it was observed that majority of cases $(47 \%)$ were above the age of 45 years and $35 \%$ of cases were in the age group of $25-45$ years and $31 \%$ cases were above the age of 55 years. Similar findings were reported by Sudhir U et al., 2011, according to his study incidence of sepsis is found in patients above 57 years. (15)

The mean age of total population was found to be statistically significant with the $p$ value 0.010 .

When cases were distributed on the basis of gender it was observed that, $76 \%$ were male and $24 \%$ were female. The male: female ratio was $3.1: 1$, it showed that the percentage of males affected with sepsis was higher as compared to females. Studies by several workers also indicated the high incidence of sepsis among males. A study by Martin et al., 2003 (16) demonstrated that sepsis was more common in men, accounting for $48.1 \%$ of cases on the average each year and men were more likely to possess sepsis than women with a mean annual relative risk of 1.28 . Another study by Todi et al., 2007 reported from a multicenter trial done at 12 centers in India that sepsis was more common in male. (17)

In the present study, during evaluating the lactate levels, it was observed that the Lactate levels were significantly high ( $p$ value $<0.0001$ ) as shown in Table 1 . According to the study done by Amit K Asati, Rajnish Gupta and D Behera (2013) high significant mean values of lactate were found among the patients of sepsis than control group $(3.13 \pm 2.86$ vs. $2.16 \pm$ 1.86 respectively, $p=0.03)$. (18)

Lactic acid is a product of anaerobic metabolism in the body. Blood lactate level is a biomarker of organ function and energy metabolism, which is related to prognosis and mortality of the patients. (19) Mainly produced by striated muscle, erythrocytes and brain tissue. Degree of its changes in plasma depends on the metabolic rate of liver and kidney in patients. (20) 
When the levels of lactate were estimated in sub-categories, then it was found that the levels of lactate were significantly increased in all three sub-groups as shown in Table 2. In this study, the overall mortality was $20 \%$ in case group. Further concluded that mortality could be attributed to age and various risk factors that are more common in that age group. Among non-survival group, $10 \%$ cases were in sepsis group, $16.22 \%$ and $33.33 \%$ were in severe sepsis and septic shock groups respectively ( $\mathrm{p}$-value-0.006).

Mean Age in patients who survived is $41.16 \pm 15.49$ as compared to patients who did not survived (45.33 \pm 10.88$)$. On the basis of survival and non- survival age mean value was found to be non-significant.

Similar pattern was observed in mean Lactate levels in the present study. Among non-survival groups, the levels were $4.20 \pm 4.56$ and $3.10 \pm 1.59$ in survival group as shown in Table 2. Study done by Zhiqiang Liu et al., 2019 illustrated the Lactate an independent prognostic predictor of mortality for patients with sepsis. (21)

According to the above study mean values of Initial, as well as, Serial lactate at all time intervals were observed to be significantly higher among non-survivors than survivors $(p$ value $=0.000$ ) according to above study. (21) In the retrospective cohort study done by Filho RR et al., (2016), lactate level more than $2.5 \mathrm{mmol} / \mathrm{L}$ was the best threshold to predict 28-day mortality among severe sepsis and septic shock patients. (22) Howell MD et al.,2007 done a prospective single-center cohort study involving 1,287 patients admitted to the Emergency Department with suspected infection showed that initial venous lactate levels between 2.5 and 4.0 $\mathrm{mmol} / \mathrm{L}$ were independently associated with an increased risk of 28-day in-hospital death. (23)

\section{CONCLUSION}

The present study was planned to assess the role of Serum lactate level as prognostic marker in patients with sepsis.

The study suggests a strong correlation between severity of sepsis and Blood Lactate levels. Moreover, the mean levels of above markers are observed higher in patients with poor outcome (non-survivors). Therefore, the study recommends screening of all patients suspected or diagnosed with sepsis for blood Lactate levels and their regular monitoring. Timely recognition of progression of sepsis can be helpful in early management of critically ill patients.

The study further recommends evaluation of association of causes of sepsis with the above markers.

\section{REFERENCES}

1. Lever A, Mackenzie I. (2007) Sepsis: definition, epidemiology, and diagnosis. BMJ.335:879-883.

2. Liu D, Su L, Han G, Yan P, Xie L. (2015) Prognostic value of procalcitonin in adult patients with sepsis: a systematic review and meta-analysis. PLoS One 5(10):1-15.

3. Dellinger RP, Levy MM, Rhodes A, Annane D, Gerlach H, Opal SM, et al. (2013) Surviving sepsis campaign: international guidelines for management of severe sepsis and septic shock. Crit Care Med.41:580-637.

4. Vincent JL, Sakr Y, Sprung CL, Ranjeri VM, Reinhart K, Gerlach H et al. (2006) Sepsis in European intensive care units: results of the SOAP study. Crit. Care Med.34(2):344-353.

5. Freund Y, Delerme S, Goulet H, Bernard M, Riou B, Hausfater P. (2012) Serum lactate and procalcitonin measurements in emergency room for the diagnosis and risk-stratification of patients with suspected infection. Biomarkers. 17:590-6.

6. Biomarkers Definitions Working Group: Biomarkers and surrogate endpoints: preferred definitions and conceptual framework. Clin PharmacolTher 2001;69:89-95.

7. Sun S, Li H, Chen J, Qian Q (2017) Lactic Acid: No Longer an Inert and EndProduct of Glycolysis. Physiology (Bethesda) 32:453-63.

8. Kraut JA,Madias NE. (2014) Lactic acidosis. NEngl J Med. 371:2309-19.

9. Vincent JL, Silva AQ, Jr LC and Taccone FS. (2016) The value of blood lactate kinetics in critically ill patients: a systematic review. Critical Care Medicine. 20(1):257:1-14

10. Suetrong B, Walley KR. (2016) Lactic acidosis in sepsis: it's not all anaerobic: implications for diagnosis and management. Chest 149:252-61.

11. David B. Sacks. Carbohydrates In: Burtis CA, Ashwood ER, editors. (1999) Tietz textbook of Clinical Chemistry. 3rd ed. Philadelphia: W.B Saunders Company; 787-790.

12. Bakker J, Gris P, Coffernils M, Kahn RJ, Vincent JL. (1996) Serial blood lactate levels can predict the development of multiple organ failure following septic shock. Am J Surg. 171(2):221-226.

13. Dellinger RP, Levy MM, Rhodes A, Annane D, Gerlach H, Opal SM et al. (2012) Surviving Sepsis campaign: international guidelines for management of severe sepsis and septic shock Crit Care Med.2013;41:580-637.

14. Long B and Koyfman A (2017). Ready for Prime Time? Biomarkers in Sepsis. emergency Medicine Clinics of North America.35.1:109-122.

15. Sudhir U, Venkatachalaiah RK, Kumar TA, Rao MY, Kempegowda P (2011) Significance of serum procalcitonin in sepsis. Indian J Crit Care Med. $\operatorname{Jan} ; 15(1): 1-5$

16. Martin GS, Mannino DM, Eaton S, Moss M (2000) The epidemiology of sepsis in the United States from 1979 through. N Engl J Med. 2003;348: 1546-54.

17. Todi S, Chatterjee S, Bhattacharyya M. (2007) Epidemiology of severe sepsis in India. Crit Care Med. 11:65.

18. Asati AK, Gupta R, Behera D. (2018) To Determine Blood Lactate Levels in Patients with Sepsis Admitted to a Respiratory Intensive Care Unit and to Correlate with their Hospital Outcomes. Int J Crit Care Emerg Med. 4(2); 1-14

19. Sankoff JD, Goyal M, Gaieski DF, Deitch K, Davis CB, Sabel AL et al. (2008) Validation of the Mortality in Emergency Department Sepsis (MEDS) score in patients with the systemic inflammatory response syndrome (SIRS). Crit Care Med.36:421-6.

20. Marty P, Roquilly A, Vallée F, Luzi A, Ferre F, Olivier F et al. (2013) Lactate clearance for death prediction in severe sepsis or septic shock patients during the first 24 hours in Intensive Care Unit:an observational study. Annals of intensive care.3(1):1-7.

21. Liu Z, Meng Z, LiY, Zhao J,Wu S, Gou S et al. (2019) Prognostic accuracy of the serum lactate level, the SOFA score and the qSOFA score for mortality among adults with Sepsis. Scandinavian Journal of Trauma, Resuscitation and Emergency Medicine 27:51;1-10

22. Filho RR, Rocha LL, Corre^a TD, Pessoa CMS, Colombo G, and Assuncao MSC (2016) Blood lactate levels cutoff and mortality prediction in sepsis-time for a reappraisal? A retrospective cohort study. Shock Nov 46 (5) 480-485

23. Howell MD, Donnino M, Clardy P, Talmor D, Shapiro NI. (2007) Occult hypoperfusion and mortality in patients with suspected infection. Intensive Care Med 33(11):1892-1899. 